\title{
Reflexiones sobre la conducta obstétrica en la embarazada con feto anencefálico
}

\author{
Manuel Sedano $\mathrm{L}^{1,2}$, Rodrigo Sedano $\mathrm{M}^{2 a}$, Rocío Sedano $\mathrm{M}^{\mathrm{b}}$, \\ Juan G Rodríguez $A^{1,2}$, Sócrates Aedo $M^{1,2}$. \\ attitude towards anencephalic fetuses
}

Pregnancy of an anencephalic fetus generates deep thoughts about its diagnosis, treatment and management of maternal risk, having in mind the irreversibility of the fetal situation. At the present moment, there are no guidelines for labor care in these cases, probably because in most developed countries in which abortion is legal, these pregnancies are interrupted earlier. In Chile, where abortion is illegal we must deal with these situations at the end of the pregnancy period. Of 35,682 labors attended at our hospital, 14 were anencephalic fetuses. In $50 \%$ of these a cesarean section was done and in one, a hysterectomy was required due to uterine inertia (Rev Méd Chile 2008; 136: 789-92).

(Key words: Anencephaly abnormalities; Ethics; Statute)

Recibido el 12 de mayo, 2008. Aceptado el 19 de mayo, 2008.

${ }^{1}$ Servicio de Obstetricia y Ginecología, Hospital Santiago Oriente Dr. Luis Tisné Brousse. Santiago de Chile. ${ }^{2}$ Departamento de Obstetricia y Ginecología (Campus Oriente), Facultad de Medicina, Universidad de Chile. Santiago de Chile.

aBecado de Obstetricia y Ginecología, Universidad de Chile.

bInterna de Medicina, Universidad de los Andes

$\mathrm{C}$ ada vez que enfrentamos la dramática situación de una madre con un embarazo con feto anencefálico, el equipo médico y cada uno de sus integrantes, no permanece ajeno a lo que la madre siente y sufre, y de una manera u otra, en un silencio reflexivo o no, se ve involucrado en la histórica búsqueda paternalista médica sobre el mejor quehacer en esta situación.

La anencefalia es el defecto más grave de las anomalías fetales; siempre es letal y ocasiona un

Correspondencia a: Dr. Manuel Sedano L.

E mail: manuelsedanol@yahoo.es óbito o la muerte neonatal temprana ${ }^{1}$. Anteayer, en el silencioso no saber y evolucionar de los embarazos con fetos anencefálicos, éramos espectadores en el momento del parto del cómo se resolvía éste: con presentaciones distócicas, ya sea de cara ${ }^{1}$ con el polo malformado o en podálica, y recién allí éramos sorprendidos por las características morfológicas del recién nacido. Ayer, en la búsqueda de las causas de un parto prematuro o de alguna complicación, fuimos ya capaces de diagnosticar que estábamos en presencia de un feto anencefálico, pero este diagnóstico se conseguía en embarazos ya avanzados, en el tercer trimestre, y utilizando métodos que en la actualidad están obsoletos, como la radiografía obstétri$\mathrm{ca}^{2}$. Hoy, con evaluaciones ecográficas precoces, 
es deseable y necesario que el diagnóstico de un feto anencefálico se realice muy tempranamente ${ }^{3}$ y con ello se ha hecho también más precoz vivir el problema de decidir la conducta obstétrica.

Algunos opinan que es necesario interrumpir el embarazo de la paciente al momento del diagnóstico, sin reflexión mayor, aunque cada caso sea diferente por ser siempre una paciente diferente. Para otros, la solución es esperar la resolución espontánea, en resguardo y protección de los riesgos que pudiesen presentarse con la interrupción del embarazo. Sin embargo, ¿en cuánto guiamos o presionamos a las pacientes a tomar decisiones?, ¿en cuánto aceptamos sus propias decisiones?, ¿en cuánto validamos el sentido común y el buen criterio en nuestras prácticas clínicas? De lo anterior se puede comprender que nadie puede arrogarse ser el portador de una verdad plena. De la interacción del equipo obstétrico con la paciente es desde donde habrá de emerger la sugerencia del "debería mejor hacer" para cada caso, nunca perdiendo de vista nuestro entorno legal particular, que finalmente regula y define los límites del quehacer.

Muchos son los puntos de apoyo para la reflexión ética sobre este tema, pero su base está en la condición funcional cerebral del feto, que es un ser irreversiblemente inconsciente, a quien nuestras acciones no podrán cambiar su condición, pero sí podemos respetarle su proceso de vida con existencia limitada dentro del vientre materno. Qué protege más a la madre o cuál opción de acción le es más favorable, es algo que se debe elegir con mucho cuidado. Cualquier intervención que adoptemos no va a beneficiar al feto, pero sí puede representar un riesgo para la madre. Los métodos utilizados para inducir un aborto o un parto prematuro, las instrumentaciones, intervenciones, anestesias y otras medicaciones, pueden generar eventos adversos maternos complejos con las consecuencias que se deriven de ellos y que, además, dejarán adicionalmente expuestos legalmente a los participantes en la atención médica. $\mathrm{Si}$ esos mismos métodos se usan en la resolución del proceso natural y se genera una complicación, ésta se enmarcará en la Lex Artis y no en la ilegalidad. Ahora bien, si evaluamos esto dentro de un sistema de salud respaldado por un marco legal que autorice el aborto en determinadas circunstancias, se debería garantizar la toma de una decisión informada competente, libre y responsable, en pleno ejercicio de la autonomía de la madre.

En la evolución espontánea o natural de estos embarazos, clásicamente se describe la aparición de polihidroamnios, distocias de presentación fetal al parto y alta mortalidad in utero ${ }^{1,4,5}$. De lo anterior, hoy no nos es posible contar con buenas estadísticas debido a que en muchos países estas situaciones se resuelven precozmente en el embarazo, en oposición a la conducta expectante que tendría 3 a 5 veces mayor riesgo de morbilidad como de mortalidad que la interrupción del embarazo en el primer trimestre ${ }^{6}$. Esta actitud activa no nos es posible observarla en nuestro medio por la legislación vigente.

En principio, en términos reales, el conflicto presenta tres opciones: la solicitud de "aborto terapéutico", la "interrupción prematura" del embarazo, o la evolución espontánea con atención del parto de iniciación espontánea (o electivo).

La caracterización clínica fetal del anencefálico, ha sido descrita en el trabajo de Besio et al, que se publica en esta Revista ${ }^{7}$, su morfología y funcionalismo lo hacen diferente, pero no lo dejan fuera de la especie, el qué hacer o no hacer no cambiará su condición y el pronóstico fetal no variará con nuestro actuar. En cuanto al pronóstico materno, si el embarazo se resuelve prematuramente con muerte fetal in utero o precozmente post aborto o post parto, ello no liberará a la madre de la eventual sensación de culpa; más bien la dejará con ella por haber participado -si lo hizo- en la decisión de interrumpir la gestación y la muerte inmediata de su recién nacido. Si buscamos proteger la salud mental materna con la interrupción del embarazo, tal vez no lo consigamos, salvo proteger la nuestra si somos partidarios de interrumpir.

Con el transcurrir de los años, las técnicas de interrupción del embarazo, que incluso costaron la vida a muchas madres, han sido perfeccionadas y con ello han disminuido sus complicaciones, pero los cuestionamientos éticos y sus alcances legales siguen vigentes.

La conducta obstétrica que se sugenía en el pasado era expectante, en espera de un parto normal, sin mayores riesgos matemos ni fetales ${ }^{8}$, pero con las advertencias de una mayor posibilidad de variedad de posición de cara, si la presentación era en encefálica 
y sugiriéndose una estricta vigilancia para el parto ante una mayor probabilidad de producirse una distocia de hombros debido al menor efecto de cuña dilatante del polo cefálico fetal y la necesidad de tener que realizar maniobras que incluyen clásicamente la fractura de la clavícula fetal o cleidotomía ${ }^{2}$.

En nuestra práctica clínica, hoy, luego del diagnóstico de anencefalia y habiendo comunicado a la madre la compleja situación encontrada a su feto, la paciente es asesorada por su obstetra tratante y por un equipo multiprofesional que incluye un psicólogo. Con la actitud acorde y uniformada de un equipo congruente, la paciente puede encontrar un respaldo que le permitirá conducirse hasta el término espontáneo, considerando incluso la opción de donar órganos de su recién nacido, al momento de nacer. Durante el tránsito hacia el término es posible que aparezcan complicaciones propias del embarazo y los riesgos propios que ello conlleva. En la experiencia recolectada por nuestro equipo, en nuestro hospital, como Centro de Referencia de malformados en la Unidad de CERPO (Centro de Referencia Perinatal Oriente), la conducta de mantener una actitud expectante, dentro del marco legal, no nos ha permitido ver complicaciones específicamente relacionadas con el embarazo o los partos de estas pacientes, realizándose su manejo y conducción de acuerdo a la historia obstétrica de cada una de ellas. Entre los años 2003 y 2007 en nuestro Servicio se han atendido 35.682 partos y de ellos 14 han sido con fetos anencéfalos. De estos 14 anencéfalos, 7 terminaron con parto por cesárea, por inducción fracasada o electivamente por su historia obstétrica (la tasa general de cesáreas en nuestro hospital es de 30\%). Todos los fetos al momento del parto, fuese vaginal o por cesárea, tuvieron presentación cefálica y hubo sólo 2 mortinatos. Con relación a las complicaciones maternas, si se considera complicación para la embarazada la cesárea, ésta se utilizó en 50\% de los casos y en uno se debió realizar histerectomía por presentar inercia uterina en el parto. Con relación a la edad de gestación al parto 7 llegaron a término, 5 se resolvieron entre las 33 y 37 semanas y sólo 2 antes de las 30 semanas ( 26 y 27 semanas, respectivamente), evolución no diferente a la observada en casos de gemelares discordantes con uno anencéfalo en que la evolución espontánea se favorece en función del feto no afectado ${ }^{5}$.
Según Besio et $\mathrm{al}^{7}$ en Sudamérica se describe una frecuencia de 11,7 casos de fetos anencefálicos por cada 10.000 nacimientos. En Estados Unidos de Norteamérica, en cambio, la incidencia sería 9,4 por 100.000 nacidos vivos, cifra que estaría subestimada por los abortos espontáneos y provocados de los fetos afectados.

En nuestro Servicio observamos una frecuencia de anencefalia de 0,04\% [CI 95\%: 0,02\% a 0,07\%] sin diferencias respecto a la frecuencia nacional informada $(0,069 \%$ [CI $95 \% 0,05 \%$ a $0,09 \%$ ] de 6,9 por 10.000 nacimientos ${ }^{9,10}$.

No pudiendo actuar en otro contexto que no sea dentro de la legalidad, es nuestra convicción que si logramos que la paciente acepte su condición y la guía hacia obtener la resolución espontánea del embarazo, o al menos alcanzar hacia el término las condiciones obstétricas aptas para su resolución, se podrá obtener en ella los mejores resultados obstétricos, que le otorguen la preservación de la salud en resguardo de su fertilidad y vida reproductiva futura. Es aceptado también que hay pacientes cuyo estado de salud previo al embarazo o por presentar patologías intercurrentes de alto riesgo, hagan recomendable la interrupción prematura del embarazo. Esto deberá proponerse a la paciente, con información sobre los riesgos médico-quirúrgicos que implican la resolución o no de su situación, para que ella tome una decisión informada.

Es de conocimiento común que ésta es una situación irreversible desde el punto de vista fetal, quien en el mejor de los casos morirá precozmente como recién nacido. Para que la madre comprenda esta evolución se requiere la participación de un equipo dispuesto a guiarla sobre la base de conocer su nivel intelectual, su familia, red de apoyo, temores y expectativas, aclaración sobre sus sentimientos de culpa y sus creencias religiosas. La situación que le corresponde vivir a este segmento de la población obstétrica, es una hoja del libro de su vida, tal vez un capítulo de su vida, que debe ser muy bien resuelto, y esta resolución deberá considerar en plenitud la decisión de la paciente, su pareja, familia, representante legal u otros vínculos validados por ella, todo en una búsqueda del concepto integral de salud para ella, sin manipulación, sin coacción y tal vez ni siquiera persuasión hacia un quehacer que siempre tendrá riesgos, que habitualmente busca resolver nuestra 
visión del problema y no la de la paciente. A ella debería concedérsele toda la información sobre la situación que va a vivir, a objeto de que pueda realizar una toma de decisión informada, en pleno ejercicio de su autonomía, ya que sería ella quien debería portar las consecuencias físicas y psíquicas, de lo que se concluya hacer y se haga en ella.

Aprendimos ayer que con actitudes patemalistas, aunque siempre inspiradas en querer ayudar, se tomaron decisiones de un hacer en estas pacientes, sin ellas participar siquiera del saber de los riesgos que involucraban las propuestas de internumpir el

\section{REFERENCIAS}

1. Cunningham FG, Leveno KJ, Bloom SL, Hauth JC, Gilstrap LCIII, WenSTROM KD. Obstetricia de Williams, Genética. Cap. 12 pag. 302, Distocias. cap. 20 pag. 507, Trastornos del líquido amniótico Cap. 21 pag. 527, vigésima segunda Edición 2006.

2. Botela Lusiá. Tratado de Ginecología "Patología Obstétrica" tomo II 1970; capítulo 25: 490.

3. Changgeng Yi Xue Za Zhi, Hsieh TT, Lo LM, Hsu JJ, ChiU TH, Lou JD ET AL. Congenital malformation in newborns. Analysis of 501 cases. Taiwan, R.O.C. 1995; 18: 9-14.

4. Holzgreve W, BeLer FK. Niños anencéfalos como donadores de órganos. Clínicas Obstétricas y Ginecológicas 1992; 4: 778.

5. Lipitz S, Meizner I, Yagel S, Shapiro I, Achiron R. Expectant management of twin pregnancies dis- embarazo. Los riesgos a que fueron sometidas para la resolución mediante abortos, incluyeron su muerte, lo que nunca guardó relación con los riesgos a que se exponían en la evolución y resolución espontánea de su situación. Hoy estos riesgos serían menores que la evolución espontánea y son la propuesta en los países desarrollados. Actualmente, muchas de nuestras reflexiones son realizadas e influidas por el marco legal en que nos encontramos para proceder en estos casos en que vamos tras la búsqueda de la autonomía de la paciente, sabiendo que dentro de ese marco no la podrán ejecutar.

cordant for anencephaly. Sch Obstet Gynecol 1995; 86: 969-72.

6. J Ayub Med Coll Abbotabad. Use of Foley's catheter and prostaglandin F-2 alpha in second trimester termination of pregnancy. Islam A. Abbasi AN, Sarwar I. 2006; 18: 35-9.

7. BesIo MR, BesIo FH. Estatuto ontológico y ético del feto anencefálico una perspectiva filosófica. Rev Méd Chile 2008; 136: 783-8.

8. Duff P. Diagnosis and management of face presentation. Obstet Gynecol 1981; 57: 105-12.

9. Nazer J, Cifuentes L, Meza M. Incidencia de las malformaciones congénitas en 10 maternidades chilenas participantes en el ECLAMC. Comparación de tres periodos (1971-1977, 1982-1988, 1989-1994). Rev Méd Chile 1997; 125: 993-1001.

10. Nazer J, Aravena T, Cifuentes L Malformaciones congénitas en Chile. Un problema emergente (periodo 1995-1999). Rev Méd Chile 2001; 129: 895-904. 